\title{
Distribution pattern following systemic mesenchymal stem cell injection depends on the age of the recipient and neuronal health
}

Claire Fabian ${ }^{1,2}$, Yahaira Naaldijk ${ }^{1,2}$, Christiane Leovsky ${ }^{1}$, Adiv A. Johnson ${ }^{3}$, Lukas Rudolph ${ }^{1}$, Carsten Jaeger ${ }^{4}$, Katrin Arnold ${ }^{1,2}$ and Alexandra Stolzing ${ }^{1,5^{*}}$

\begin{abstract}
Background: Mesenchymal stem cells (MSCs) show therapeutic efficacy in many different age-related degenerative diseases, including Alzheimer's disease. Very little is currently known about whether or not aging impacts the transplantation efficiency of MSCs.

Methods: In this study, we investigated the distribution of intravenously transplanted syngeneic MSCs derived from young and aged mice into young, aged, and transgenic APP/PS1 Alzheimer's disease mice. MSCs from male donors were transplanted into female mice and their distribution pattern was monitored by PCR using Y-chromosome specific probes. Biodistribution of transplanted MSCs in the brains of APP/PS1 mice was additionally confirmed by immunofluorescence and confocal microscopy.

Results: Four weeks after transplantation into young mice, young MSCs were found in the lung, axillary lymph nodes, blood, kidney, bone marrow, spleen, liver, heart, and brain cortex. In contrast, young MSCs that were transplanted into aged mice were only found in the brain cortex. In both young and aged mouse recipients, transplantation of aged MSCs showed biodistribution only in the blood and spleen. Although young transplanted MSCs only showed neuronal distribution in the brain cortex in young mice, they exhibited a wide neuronal distribution pattern in the brains of APP/PS1 mice and were found in the cortex, cerebellum, hippocampus, olfactory bulb, and brainstem. The immunofluorescent signal of both transplanted MSCs and resident microglia was robust in the brains of APP/PS1 mice. Monocyte chemoattractant-1 levels were lowest in the brain cortex of young mice and were significantly increased in APP/PS1 mice. Within the hippocampus, monocyte chemoattractant-1 levels were significantly higher in aged mice compared with younger and APP/PS1 mice.
\end{abstract}

Conclusions: We demonstrate in vivo that MSC biodistribution post transplantation is detrimentally affected by aging and neuronal health. Aging of both the recipient and the donor MSCs used attenuates transplantation efficiency. Clinically, our data would suggest that aged MSCs should not be used for transplantation and that transplantation of MSCs into aged patients will be less efficacious.

Keywords: Mesenchymal stem cells, Biodistribution, Systemic injection, Aging, Alzheimer's disease

\footnotetext{
* Correspondence: A.Stolzing@lboro.ac.uk

${ }^{1}$ Interdisciplinary Centre for Bioinformatics (IZBI), University of Leipzig,

Leipzig, Germany

${ }^{5}$ Centre for Biological Engineering, Wolfson School, Loughborough

University, Loughborough, UK

Full list of author information is available at the end of the article
} 


\section{Background}

Mesenchymal stem cells (MSCs) are a heterogenic mix of progenitor and stem cells that can differentiate into various mesenchymal tissues, including cartilage, bone, and adipose tissue [1]. Transplantation of MSCs has been investigated for a variety of diseases and many of these approaches have already entered clinical trials [2]. MSCs are often administered systemically for clinical application and systemic delivery of MSCs has been performed for a variety of ailments, including osteogenesis imperfecta [3], bone defects [4], diabetes [5], myocardial infarctions [6], multiple sclerosis [7], and arthritis [8]. The underlying regenerative mechanism of action seems to be related to the immune-modulatory, anti-inflammatory, and anti-fibrotic activity of MSCs $[9,10]$.

The success of a given MSC transplantation therapy may depend on a variety of factors, such as whether or not they were pre-incubated with protective compounds $[11,12]$ or under specific environmental conditions [13]. Another key factor underlying MSC transplantation efficiency may be the ability of MSCs to localize, or migrate, into target tissues of interest [14]. Intravenously transplanted MSCs have been found in very low frequencies in different organs using various labelling and tracking methods, including fluorescently labelled cells, xenogeneic transplantation, and sex-linked chromosome detection tracked by PCR [15-19]. Twenty-four hours after intravenous transplantation, MSCs are mostly found in the lung, liver, kidney, skin, thymus, lymph node, and gut with $1-2.7 \%$ of transplanted cells homing to these different organs in young animals [20]. MSCs first accumulate in the lung 24-48 hours after transplantation but can be found later in the liver, kidney, spleen, and other organs, particularly those showing injury [21]. Multimodal MRI nanoparticles with enhanced near-infrared fluorescence have been used recently to perform in-vivo imaging of human adipose-derived stem cells in an Alzheimer's disease mouse model [22]. Cells administered via tail-vein injection were observed in the tail, body, and brain of Alzheimer's disease mice up to 10 days after transplantation (with the strongest signal at day 3), but not in the brains of wild-type (WT) controls [22]. Post-mortem examination of organs revealed weak fluorescent signals in WT brain tissue, suggesting that some cells are able to cross the blood-brain barrier (BBB) in young animals. However, the strongest signals were observed in the Alzheimer's disease mouse brains, which the authors attribute to leakage of the BBB brought about as a result of neurological disease. In addition, human adipose-derived stem cells had transmigrated to the gastrointestinal tract, kidney, liver, and bladder of all injected mice [22].

MSCs mediate numerous therapeutic effects by promoting repair directly via differentiation into critical cell types or indirectly through the secretion of substances and the activation of endogenous mechanisms [23]. In order to mediate such beneficial effects, MSCs must first home or migrate to a specific site of injury or damage. MSC migration is therefore thought to play a paramount role in the remedial process [23]. Migration of MSCs is subject to extensive regulation $[13,23,24]$ and existing evidence suggests that MSCs migrate to specific organs in a manner similar to leukocytes. They adhere to endothelial cells in the vascular system and transmigrate across the vascular endothelium [25] towards injured and inflamed tissues [26, 27]. MSCs home to areas of injury along a chemokine gradient and several chemokines have been identified which affect MSC migration, including stromal cell derived factor 1 alpha, CXC chemokine receptor type 4, transforming growth factor beta 1, interleukin 1 beta, and tumour necrosis factor alpha [24, 28, 29]. Many of these specific regulatory factors have been shown to also affect the expression of chemokines and selectins in MSCs [13, 30-32].

Although conflicting reports exist in the literature as to whether or not MSC migration is affected by aging [13, 33], aging is known to detrimentally affect MSC functionality [1]. More broadly, aging is known to exert negative effects on stem cells and progenitor cells [34-36]. Aging increases the susceptibility of MSCs to damaging agents like reactive oxygen species, disrupts cell population dynamics, diminishes therapeutic efficacy, and mediates other harmful effects [1, 37-39]. While numerous studies have uncovered aspects of MSC aging, it remains to be determined whether or not aging affects MSC transplantation efficiency. Moreover, further data are required to clarify whether or not MSC migration and engraftment is affected in vivo by aging. To better elucidate these questions, we assessed MSC biodistribution in vivo in various mouse organs following transplantation with young or old MSCs into young, old, or diseased animals.

\section{Methods \\ Animals}

C57Bl/6 mice as a source for bone marrow were obtained from the University of Leipzig or Charles River. The GFP transgenic mice were from the Paul Flechsig Institute for Brain Research, University of Leipzig, and the transgenic mice overexpressing human amyloid precursor protein $\left(\mathrm{APP}_{\mathrm{KM} 670 / 671 \mathrm{NL}}\right)$ and presenilin-1 $\left(\mathrm{PS}_{\mathrm{L} 166 \mathrm{P}}\right)$ under Thy-1 promoter control were obtained from University of Leipzig (Prof. Bechmann).

\section{MSC preparation}

After isolation, bone marrow from tibiae and femurae were harvested as described previously [40] and cultured in DMEM 1×, low glucose (Gibco) with GlutamaxI (Gibco), 10\% fetal calf serum (Biochrome), and 1\% penicillin/streptomycin (Gibco) for the isolation of adherent 
MSCs. Briefly, mouse bone marrow cells were obtained by centrifugation from tibiae and femurae and were cultured according to the method of Dobson et al. [41]. MSCs were isolated by the method of Sekiya et al. [42]. MSCs were passaged when they were at 70\% confluency using trypsin (0.25\% trypsin-EDTA; Gibco). Like we have done previously [43], fluorescence-activated cell sorting was used to confirm the identity of MSCs by assessing specific markers (e.g., CD11b ${ }^{-}, \mathrm{CD} 45^{-}, \mathrm{CD} 44^{+}$, $\mathrm{CD}^{+} 0^{+}$). Similarly to earlier studies $[1,13,43]$, mesodermal lineage differentiation was also performed to confirm successful MSC isolation and culturing.

\section{MSC transplantation}

To set up a sex-mismatched transplantation, $1 \times 10^{6}$ MSCs (passages 2-3) from male donors were transplanted into female recipients by tail-vein injection. MSCs were isolated either from young mice (2-3 months old) or from aged mice (12-13 months old). Recipient mice were either young (3 months old) or old (13-21 months old). Older APP/PS1 mice (12-15 months old) also received young MSCs. Twenty-eight days after transplantation, the mice were sacrificed and the organs isolated for analysis. Three to five mice were used for each experimental group. Three male mice were also transplanted with $1 \times 10^{6}$ bonemarrow derived MSCs from female GFP-transgenic mice (3 months old) and were sacrificed for histological analysis 28 days after transplantation.

\section{Preparation of tissue}

For genomic DNA (gDNA) isolation, mice were sacrificed 28 days after transplantation and transcardially perfused with $0.9 \% \mathrm{NaCl}$. Peripheral organs (lung liver, kidney, heart, lymph nodes, bone marrow, spleen) were removed, as well as the brain which was divided into five regions (hippocampus, cortex, cerebellum, brainstem, olfactory bulb). The tissue was mechanically homogenized in peqGOLD TriFast ${ }^{\mathrm{m}}$ (PeqLab) and stored at $-80{ }^{\circ} \mathrm{C}$ until further use.

For histology, mice were sacrificed after 28 days and perfused transcardially with $0.9 \% \mathrm{NaCl}$ followed by $4 \%$ paraformaldehyde and $0.1 \%$ glutaraldehyde in $0.1 \mathrm{M}$ phosphate buffer ( $\mathrm{pH}$ 7.4). Brains were removed and immersion-fixed overnight in the same fixative at $4{ }^{\circ} \mathrm{C}$. Brains were cryoprotected in $30 \%$ sucrose in $0.1 \mathrm{M}$ phosphate buffer ( $\mathrm{pH}$ 7.4) with $0.1 \%$ sodium azide, cut into $40 \mu \mathrm{m}$ slices with a cryomicrotome in the frontal plane, and collected in $0.1 \mathrm{M}$ phosphate buffer (pH 7.4) with $0.1 \%$ sodium azide.

\section{gDNA isolation}

gDNA was isolated using peqGOLD TriFast ${ }^{\mathrm{mm}}$ (PeqLab) according to the manufacturer's instructions. After isolation, gDNA was fractionated through an injection needle (size 2; Braun) using a 0.01-1 ml syringe (Braun). gDNA was then either further purified using a gDNA clean-up kit (Macherey-Nagel) or purified and enriched using a gDNA clean-up XS kit (Macherey-Nagel). All kits were used according to the manufacturer's instructions.

\section{RNA isolation and quantitative RT-PCR}

RNA was extracted from organs using peqGOLDTrifast ${ }^{\mathrm{tm}}$ reagent according to the manufacturer's instructions (302040; PeqLab) and treated with DNaseI (EN0521; Life Technologies). This was followed by cDNA synthesis using SuperscriptIII reverse transcriptase (18080085; Life Technologies) and Oligo (dT)18-Primers (SO132; Thermo Scientific) at $50^{\circ}$ $\mathrm{C}$ for 1 hour. cDNA was used as PCR template in a 1:10 dilution and each sample run in triplicate. Quantitative PCR was performed using Express SYBR GreenER qPCR Supermix Universal (1178401 K; Life Technologies), additional 1× SybrGreen (S-7567; Life Technologies), and $0.2 \mu \mathrm{M}$ primer each on the DNA engine Opticon2 (Biorad) (see Table 1) with the following cycle conditions: primary denaturation at $95{ }^{\circ} \mathrm{C}$ for $3 \mathrm{~min}, 35$ cycles for $30 \mathrm{~s}$ at $95{ }^{\circ} \mathrm{C}, 30 \mathrm{~s}$ at $60{ }^{\circ} \mathrm{C}$, and $30 \mathrm{~s}$ at $72{ }^{\circ} \mathrm{C}$, followed by fluorescence measurement. Absolute quantification was performed for every single gene with three technical repeats per sample. Serial dilutions of plasmid controls with known molecule concentrations were used as positive control and to generate standard curves for the PCR. Expression of target genes was normalized using 36B4 (large ribosomal protein P0, RPLP0) as a reference gene.

\section{Cell-tracking PCR}

Male gDNA was tracked by PCR using Y-chromosome specific primers (5' -CATGGAGAGCCACAAGCTAACCA-3' and $5^{\prime}$-GTCCCAGCATGAGAAAGATTCTTCC-3'). Up to four copies of Y-chromosomes can be detected in the background of up to $50 \mathrm{ng}$ gDNA. The PCR mix contained $0.2 \mu \mathrm{M}$ primers, $0.2 \mathrm{mM}$ dNTPs (Thermo Scientific), $2.8 \mathrm{mM} \mathrm{MgCl}_{2}$ (Life Technologies), $1 \times$ buffer without $\mathrm{MgCl}_{2}$ (Life Technologies), and $1 \mathrm{U}$ platinum taq polymerase (Life Technologies). gDNA ( $5 \mu \mathrm{l}$ ) was added to the mix as a template. As a positive control, $5 \mu \mathrm{gDNA}$ isolated from male mouse brain tissue was used (concentration: $0.55 \mathrm{ng} / \mathrm{ml}$ in TE buffer, $\mathrm{pH}$ 8.0) instead of the sample gDNA. The single copy gene 36B4 (5' -CCGTGTGAGGTCACTGTGCCAGCTC-3' and 5'-GCCCAAAGCCTGGA AGAAGGAGGTC-3') was used as reference gene. PCR

Table 1 PCR primers

\begin{tabular}{lllll}
\hline Name & Gene & $\begin{array}{l}\text { Sense primer } \\
\left(5^{\prime}-3^{\prime}\right)\end{array}$ & $\begin{array}{l}\text { Antisense primer } \\
\left(5^{\prime}-3^{\prime}\right)\end{array}$ & $\begin{array}{l}\text { NCBl accession } \\
\text { number }\end{array}$ \\
\hline 36 B4 & $\begin{array}{l}\text { Ribosomal } \\
\text { protein }\end{array}$ & $\begin{array}{l}\text { CCGTGTGA } \\
\text { GTCACTGTG }\end{array}$ & $\begin{array}{l}\text { GCCCAAGG } \\
\text { CTGGAGA }\end{array}$ & NM_007475.5 \\
& CCAGCTC & AGGAGGTC & \\
RPLPO & & & \\
MCP-1 & $\begin{array}{l}\text { Transcription } \\
\text { regulatory } \\
\text { protein }\end{array}$ & GCAGTAATC & GATCCCGTC & L13763.1 \\
& CCACCAGC & CTCATCCAG & \\
MCPOU 1) & & ACTTGG & \\
\hline
\end{tabular}


was performed in a thermocycler $\mathrm{T}$ professional (Biometra) with a primary 3-min denaturation step at $95{ }^{\circ} \mathrm{C}$ followed by 35 cycles: $30 \mathrm{~s}$ at $95^{\circ} \mathrm{C}, 30 \mathrm{~s}$ at $60{ }^{\circ} \mathrm{C}$, and $30 \mathrm{~s}$ at $72{ }^{\circ} \mathrm{C}$, with a final 3-min elongation step at $72{ }^{\circ} \mathrm{C}$. The PCR products were verified by agarose gel electrophoresis.

\section{Immunohistochemistry}

Immunohistochemistry was performed with free-floating sections. Brain slices were washed once with PBS containing 0.05\% Tween20 (PBS-T) (P2287-100ML; Sigma-Aldrich) and treated with 60\% methanol (VWR Prolabo) for 1 hour. Sections were incubated in blocking solution consisting of PBS-T plus 2\% bovine serum albumin (11930; Serva), 0.3\% milk powder (T145; Roth), and 0.5\% donkey normal serum (017-000-001; Jackson Immuno Research) for $30 \mathrm{~min}$ prior to incubation with the primary antibody. Immunofluorescence staining for microglia was performed with rabbit antiIba-1 (1:500; WAKO) in blocking solution overnight at $4{ }^{\circ} \mathrm{C}$, followed by secondary antibody donkey-anti-rabbit $\mathrm{Cy} 3$ (1:250, 711-165-152; Dianova) for 1 hour. Finally, slices were washed three times and incubated for $10 \mathrm{~min}$ with DAPI (1:10,000; Sigma) at $4{ }^{\circ} \mathrm{C}$ and washed once. For immigrated $\mathrm{GFP}^{+}$MSCs, no immunohistochemical enhancement was used. Slices were mounted on glass slides, dried, and coverslipped with entellan in toluol (108323; Merck).

\section{Microscopy and image processing}

Tissue sections were examined with the Keyence BZ-9000 microscope (Keyence Corporation, USA). Fluorescence labelling was examined with a Zeiss confocal laser scanning microscope (LSM 510; Zeiss, Jena, Germany). For imaging of GFP (green fluorescence), an argon laser with 488-nm excitation was used and emission from Cy2 was recorded at $510 \mathrm{~nm}$ with a low-range band pass filter (505-550 nm). For secondary Cy3-labelling (red fluorescence), a helium-neon laser with 543-nm excitation was used and emission from Cy3 at $570 \mathrm{~nm}$ was detected applying a high-range band pass (560-615 nm). For DAPI labelling, a 405-nm diode laser was used. Photoshop CS2 (Adobe Systems, Mountain View, CA, USA) was used to process the images with minimal alterations to the brightness, sharpness, colour saturation, and contrast.

\section{Statistical analysis}

Data are presented as the mean of the samples and standard deviation as the standard error of the mean (SEM). Statistical analysis was performed using SigmaPlot 11.0 software (Systat Software Inc.) using one-way ANOVA. $p<0.05$ was considered statistically significant.

\section{Results}

\section{Post-transplantation health and MSC phenotyping}

None of the mice receiving MSCs died or showed any pathological changes post transplantation. All MSCs used for transplantation were at culture passages $2-3$ and exhibited tri-lineage differentiation potential into adipocytes, chondrocytes, and osteocytes (Additional file 1: Figure S1). MSCs transplanted were positive for CD29, CD44, CD73, CD105, and CD106 and were negative for CD45 and CD11 (Additional files 2 and 3: Figure S2 and S3).

\section{Biodistribution of systemically transplanted young MSCs into young and old mice}

MSCs from young male donors were transplanted into young and old female recipients via tail-vein injection. As described in the Methods, Y-chromosome PCR analysis was then performed to assess the biodistribution of MSCs in isolated organs and tissues post mortem. Following a tail-vein injection, young transplanted MSCs were found in the lung, axillary lymph nodes, blood, kidney, bone marrow, spleen, liver, heart, and brain cortex of young mice (Fig. 1a). In contrast, male gDNA was only present in the brain cortex of old mice following transplantation with young MSCs (Fig. 1a).

\section{Biodistribution of systemically transplanted old MSCs into young and old mice}

When aged MSCs were transplanted into young or aged mice, transplanted MSCs were only found in the blood and spleen (Fig. 1b). Although more MSCs were found in the spleen of young recipient mice, more MSCs were present in the blood of aged recipient mice (Fig. 1b). When young MSCs were transplanted into young and old mice, biodistribution in the brain was exclusive to the cortex (Fig. 1a). Conversely, aged MSCs were not found in any neuronal tissue post transplantation into either young or old mice (Fig. 1b). In comparison with young MSCs transplanted into young mice (Fig. 1a), aged MSCs transplanted into young mice showed a markedly decreased biodistribution (Fig. 1b).

\section{Biodistribution of systemically transplanted young MSCs into old APP/PS1 mice}

In $\mathrm{C} 57 \mathrm{Bl} / 6$ recipients, transplanted young MSCs showed neuronal distribution in the brain cortex (Fig. 1a) while transplanted old MSCs were not found in any brain tissues (Fig. 1b). In contrast, young MSCs which were transplanted into aged APP/PS1 mice were predominantly found in neuronal tissues. In addition to being distributed in the lung, bone marrow, and kidney, transplanted MSCs in these mice were found in all isolated brain parts, including the cortex, cerebellum, hippocampus, olfactory bulb, and brainstem (Fig. 1c). The strongest signals were found in the hippocampus and brainstem. APP/PS1 mice represent a mouse model of Alzheimer's disease and, in this murine model of neuronal degeneration, MSCs transplanted via tail-vein injections homed preferentially into the brain. 

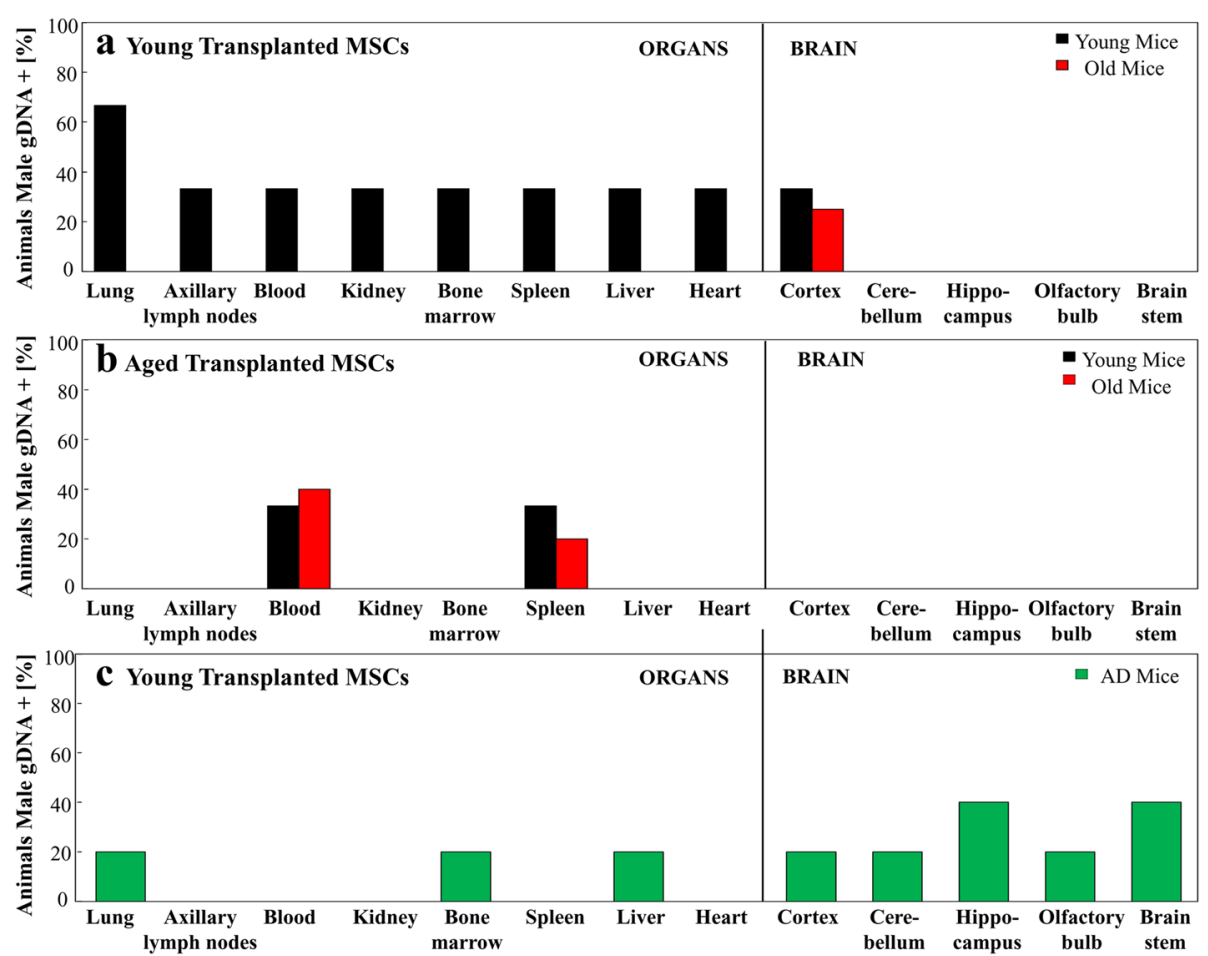

Fig. 1 Biodistribution of young and old MSCs transplanted into young, old, and APP/PS1 mice. MSCs derived from young or old male mice were transplanted into young, old, and Alzheimer's disease female mice via tail-vein injections. Percentage of male gDNA in organs was assessed and quantified 28 days after transplantation. (a) Young MSCs were intravenously transplanted into either young $(n=3)$ or old $(n=4)$ recipient mice. Young MSCs were found in the lungs, axillary lymph nodes, blood, kidney, bone marrow, spleen, liver, heart, and brain cortex of mice. In contrast, young MSCs were only found in the brain cortex of old mice. (b) Aged MSCs were transplanted into young $(n=3)$ and old $(n=5)$ mice. Biodistribution of aged MSCs was exclusive to the blood and spleen. (c) Young MSCs were injected into the tail veins of APP/PS1 Alzheimer's disease (AD) mice $(n=5)$. Biodistribution was found in the cortex, cerebellum, hippocampus, olfactory bulb, brain stem, liver, bone marrow, and lung. gDNA genomic DNA, MSC mesenchymal stem cell

To further confirm that MSCs were indeed homing to the brain following a tail-vein injection, we employed immunofluorescence staining in addition to our Y-chromosome PCR analysis. $\mathrm{GFP}^{+} \mathrm{MSC}$ from young GFP-positive mice were transplanted into aged APP/PS1 mice via tail-vein injection. Immunofluorescent staining for nuclei (DAPI) and Iba-1, a marker of microglia [44], was then performed on frontal sections. In correspondence with the Y-chromosome PCR results, immigrated $\mathrm{GFP}^{+}$MSCs were found in all brain regions, especially in the cortex and hippocampus. $\mathrm{GFP}^{+}$ MSCs appeared strongly attracted to inflammation sites and seemed to be integrated into the network of resident microglia (Fig. 2). A representative, low-magnification image is also included to highlight the reliability of this staining pattern (Additional file 4: Figure S4).

\section{Expression of monocyte chemoattractant protein-1 in young, aged, and APP/PS1 mouse brains}

Monocyte chemoattractant protein-1 (MCP-1) is known to regulate the function and behaviour of MSCs $[45,46]$. Using PCR, we measured MCP-1 gene levels in the hippocampus and cortex of young, aged, and APP/PS1 mice (Fig. 3). We found that, in the hippocampus, MPC-1 levels were significantly higher $(p<0.001)$ in aged mice compared with $M C P-1$ levels in young and APP/PS1 mice (Fig. 3a). In the cortex, $M C P-1$ expression was the lowest in young mice (Fig. 3b). $M C P-1$ expression was highest in APP/PS1 mice and was significantly greater $(p<0.001)$ than $M C P-1$ expression in both young and aged mice (Fig. 3b).

\section{Biodistribution overview}

The results from the biodistribution experiments are summarized visually in Fig. 4. It is especially lucid that aging impacts transplantation efficiency when the data are presented in this tabular manner, because no young transplanted MSCs were found to home in any organ except the brain in any of the four aged mice tested (Fig. 4). Conversely, young MSCs were found in at least one organ in every young mouse recipient. Additionally, the overall engraftment was clearly lower in MSCs from aged donors compared with MSCs from young donors (Fig. 4). That MSCs migrated to various regions of the brain (young MSCs to the cortex of young and aged mice, and young MSCs to the cortex, cerebellum, 

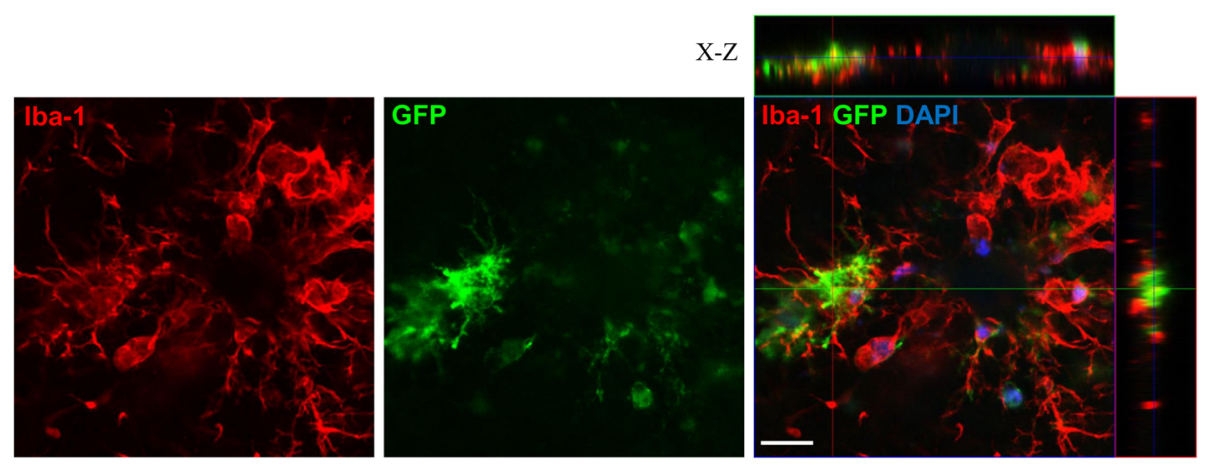

Y-Z

Fig. 2 Migration of transplanted MSCs into the brain of APP/PS1 mice. Intravenously transplanted GFP ${ }^{+}$MSCs were able to migrate into the brain parenchyma. GFP ${ }^{+}$MSCs (green, centre panel) were found in association with activated microglia (Iba-1; red, left panel), but only partly showing overlap with Iba-1 immunoreactivity (right panel). In the merged image (orthoview based on stack analysis), Iba-1 and GFP are shown in combination with nuclei (DAPI, blue) and, in addition to the X-Y image, orthogonal X-Z and Y-Z projections are shown. Scale bar: $20 \mu \mathrm{M}$ (Colour figure online)

hippocampus, olfactory bulb, and brain stem of APP/ PS1 mice) is also very apparent (Fig. 4).

\section{Discussion}

In our previous work, we demonstrated that aging detrimentally affects intravenously or intranasally transplanted in-vitro differentiated microglia derived from mouse bone marrow [47]. Specifically, we showed that transplanted microglia from young donors migrated to the brain in both young and old recipients while transplanted microglia from older donors failed to exhibit migration into the brain [47]. In the present work, we corroborate and expand upon this prior work [47] by presenting evidence that aging substantially hinders the transplantation efficiency of MSCs.
Although young MSCs migrated to nine organs when transplanted into young mice, young MSCs only migrated into a single organ in old mice. This result demonstrates that, even when young, robust MSCs are used for transplantation, the age of the recipient drastically affects transplantation efficiency and post-transplantation migration. Aspects of senescence in the elderly recipients must therefore be preventing the efficient migration or survival of MSCs. We recently showed that cytokines, growth factors, and $\mathrm{O}_{2}$ concentration affect MSC migration [13]. These and other age-dependent regulatory factors $[35,48]$ are probably anomalous in elderly mice and this dysregulation might be responsible for the restricted immigration of MSCs into the brain.

We further investigated how effectively intravenously transplanted aged MSCs would distribute in both young
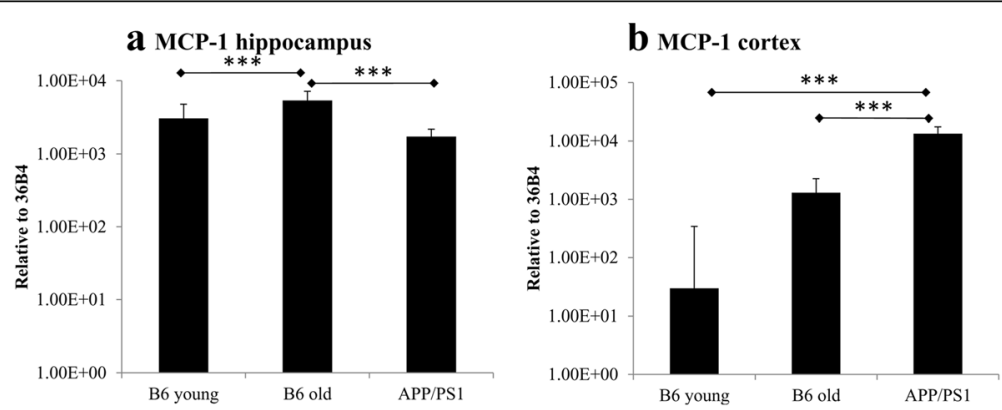

Fig. 3 Monocyte chemoattractant protein-1 (MCP-1) levels in the hippocampus and cortex of young, aged, and APP/PS1 mice. Using PCR, the levels of the chemokine and MSC regulator MCP-1 were quantified in the hippocampus and cortex of young, aged, and APP/PS1 mice without cell treatment. a Compared with young and APP/PS1 mice, MCP-1 levels were significantly increased in the hippocampus of old mice. $\mathbf{b}$ Within the brain cortex, young mice showed the lowest levels of $M C P-1$ expression. Compared with both young and old mice, $M C P-1$ levels were significantly increased in the brain cortex of APP/PS1 mice. ${ }^{* *} p<0.001$ 

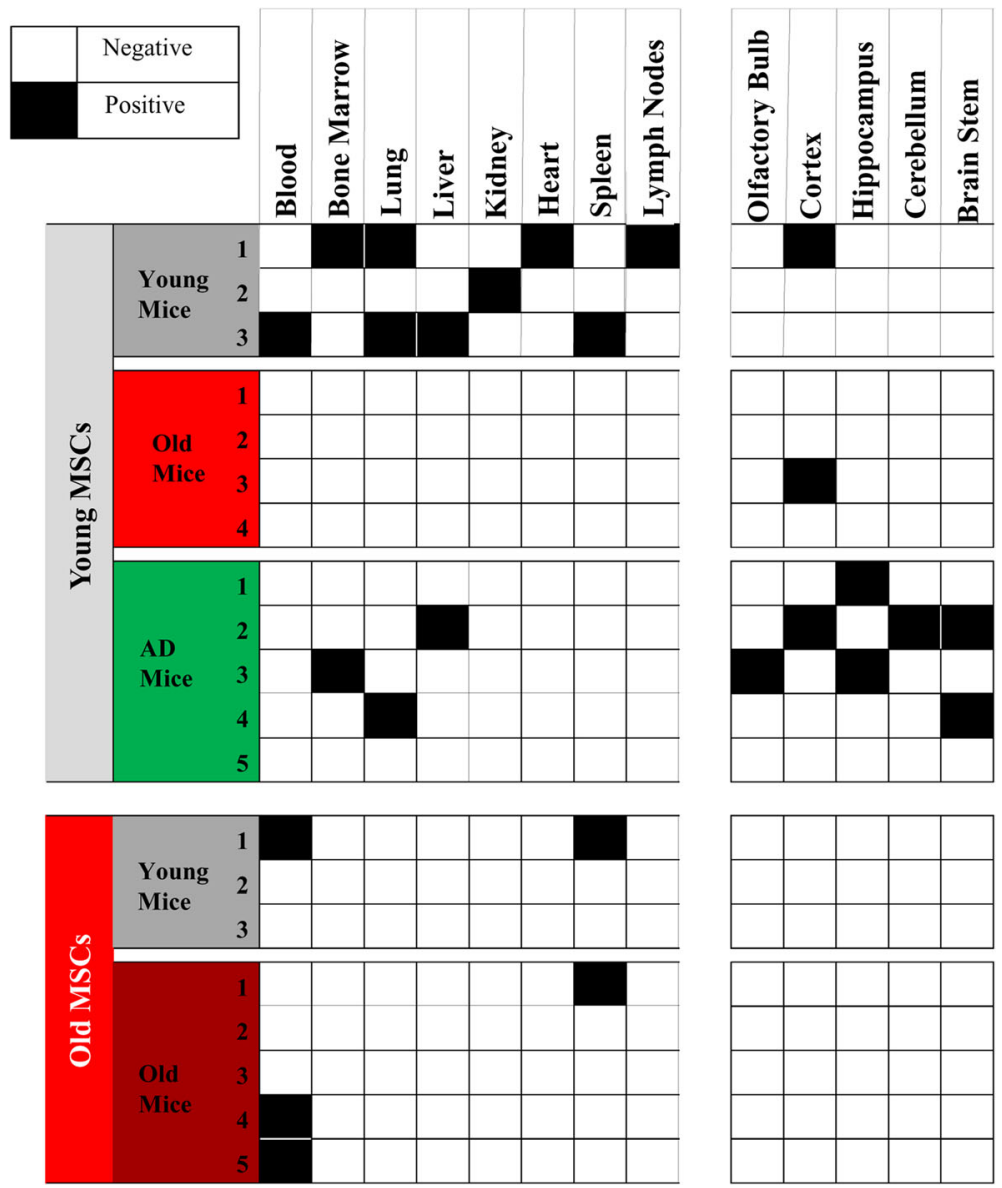

Fig. 4 Biodistribution overview. Tabular biodistribution summary for each of the mice used for the transplantation experiments. AD Alzheimer's disease, MSC mesenchymal stem cell

and old mice. Consistent with our findings regarding transplantation efficiency of young MSCs into aged mice, we found that aged MSCs revealed a dramatically reduced biodistribution in both young and aged recipients and were only found in two tissues-the blood and the spleen. Here, more young MSCs were found in the spleen of old mice and more old MSCs were found in the blood of old mice. We postulate that young MSCs were more capable of migrating into the spleen tissue while older MSCs were less able to leave the blood and home into this organ. Because this difference was not statistically significant, more research is required to determine whether this disparity is invariable.

These data indicate that, in addition to the age of the recipient host affecting MSC biodistribution, the age of the used MSCs themselves affects distribution into various tissues. Aging has been reported previously to detrimentally affect the ability of MSCs to mediate wound healing and vascular support [37]. Moreover, work by Bustos et al.
[33] suggests that aging may impair the migratory and anti-inflammatory abilities of MSCs. Our data substantiate these and other claims that aging detrimentally affects MSC functionally. We add novel in-vivo data showing that the deleterious effects of aging significantly attenuate MSC biodistribution post transplantation.

Unlike aged C57Bl/6 mice, aged APP/PS1 mice showed a broad biodistribution following transplantation with young MSCs, with a preferential attraction towards the brain. Young MSCs were found in each of the examined brain areas (cortex, cerebellum, hippocampus, olfactory bulb, brainstem) following transplantation. Because some controversy still exists on whether or not MSCs can cross the BBB [49], we verified biodistribution in the brain by both Ychromosome PCR and immunofluorescence detection of $\mathrm{GFP}^{+}$MSCs. Our mice were not irradiated and not immune suppressed, which confirms that MSCs can cross the $\mathrm{BBB}$ in untreated, young, and aged mice. Concerning the APP/PS1 mice, the BBB is known to be affected in 
neurodegenerative disorders like Alzheimer's disease. BBB breakdown due to disrupted tight junctions, altered transport processes, and inflammatory effects have been reported to underlie neurodegenerative diseases [50]. These factors would make it notably easier for MSCs to infiltrate the brain.

The preferential biodistribution in the brain of recipient APP/PS1 mice compared with recipient young and aged $\mathrm{C} 57 \mathrm{Bl} / 6$ mice might also be in part due to differences in MCP-1 levels. In the brain cortex, APP/PS1 mice showed significantly higher $M C P-1$ levels compared with old and young mice. Because MCP-1 is known to stimulate MSC migration [51], this increased $M C P-1$ signal in the brain cortex of APP/PS1 mice might be a factor that attracts more MSCs into the brain. A confounding factor that does not support this hypothesis is that, although $M C P-1$ levels were also increased in older mice compared with young mice, older mice recipients showed a significantly reduced biodistribution. It is important to note, however, that this difference in MCP-1 levels between older and younger mice was not statistically significant.

Another paradoxical factor is that, in the mouse hippocampus, $M C P-1$ levels were lowest in APP/PS1 mice and highest in aged mice. Aged mice exhibited significantly higher $M C P-1$ levels than both young and APP/PS1 mice. One possible explanation for this is that amyloid plaques in this Alzheimer's disease mouse model are more abundant in the cortex than in the hippocampus [52], which could explain why $M C P-1$ levels were significantly increased in the brain cortex but not in the hippocampus of APP/PS1 mice. Amyloid- $\beta$ can activate microglia, which in turn has been reported to increase MCP-1 production in rodents and humans [53]. Another possibility is that MCP-1 levels do not discernibly affect biodistribution and that the primary factor increasing neuronal distribution in $\mathrm{APP} / \mathrm{PS} 1$ is disruption of the BBB due to neurodegeneration. Follow-up research is required to understand the detailed mechanisms underlying the observed differences in biodistribution between young, old, and APP/ PS1 mice.

\section{Conclusions}

In sum, we demonstrate in vivo that MSC biodistribution post transplantation is detrimentally affected by aging and neuronal health. Aging of both the recipient and the donor MSCs used attenuates transplantation efficiency. Clinically, our data would suggest that aged MSCs should not be used for transplantation and that transplantation of MSCs into aged patients will be less efficacious. Further studies are warranted to identify novel therapeutic strategies to improve biodistribution of MSCs in aged hosts. Additional studies are also warranted to see whether aging affects post-transplantation biodistribution in other disease models and to determine whether young and old MSCs preferentially home to organs of interest in aged versus young animals.

\section{Additional files}

\begin{abstract}
Additional file 1: Figure S1. showing mesodermal lineage differentiation of bone-marrow-derived MSCs. Bone-marrow-derived MSCs were differentiated in vitro under adipogenic (a), osteogenic (b), or chondrogenic (c) conditions. Verification of the differentiation was done by qualitative analysis: adipogenesis shown by staining lipid vesicles with Oil red-O (a), osteogenesis shown by staining alkaline phosphatase with Fast Red (b), and chondrogenesis shown by staining sulphated proteoglycans typical for extracellular matrix composition with Alcian Blue under acidic conditions.
\end{abstract}

Additional file 2: Figure S2. showing cell marker panels of Sca1, CD73, CD105, CD29, and CD45 in bone-marrow derived MSCs. A cell marker panel was performed via FACS on Bl6, bone-marrow-derived MSCs at passage 3 . P2-Q4 represents the negative quadrant in the bottom left (purple). P2-Q1 represents the positive quadrant in the upper left (green). (TIF 1142 kb)

Additional file 3: Figure S3. showing cell marker panels of CD11b, CD106, and CD44 in bone-marrow-derived MSCs. A cell marker panel was performed via FACS on BI6, bone-marrow-derived MSCs at passage 3. P2-Q4 represents the negative quadrant in the bottom left (purple). P2-Q1 represents the positive quadrant in the upper left (green). Bar graph (bottom right) and textual table (bottom left) included to summarize the overall results of the FACS experiments. (TIF 831 kb)

Additional file 4: Figure S4. showing a low-magnification image of transplanted MSCs in the brain of APP/PS1 mice. Representative low-magnification image showing the migration of transplanted GFP-positive MSCs (green) into the brains of APP/PS1 mice. GFP-expressing MSCs were found in association with activated microglia (lba-1, red). Sections were stained with DAPI to highlight nuclei as a positional marker. Scale bar. $50 \mu \mathrm{M}$. (TIF 4445 kb)

Abbreviations

BBB: Blood-brain barrier; MCP-1: Monocyte chemoattractant protein-1; MSC: Mesenchymal stem cell; WT: Wild type

\section{Acknowledgements}

The authors are grateful for the funding provided by the German Federal Ministry of Education and Research.

Funding

The work presented in this article was made possible by funding from the German Federal Ministry of Education and Research (BMBF 031A575C).

Availability of data and materials

All data generated or analysed during this study are included in this published article and its supplementary information files.

\section{Authors' contributions \\ AS, YN and CJ were responsible for the transplantation overview. YN, CJ, LR and $\mathrm{CL}$ carried out bone marrow isolation, MSC cultivation, transplantation, perfusion, organ isolation, and histology analysis. CJ performed confocal laser scanning microscopy analysis. CL carried out DNA isolation, performed data analysis, and helped summarize the data. CF was involved in DNA isolation, PCR analysis, primer design, and data analysis. AS was responsible for data analysis and the overall design of the project. AAJ, AS, and $\mathrm{YN}$ wrote the manuscript. AAJ also assisted with data analysis. KA performed experiments and collected data to characterize the mesenchymal stem cells used in the study. All authors read and approved the final manuscript.}

Competing interests

The authors declare that they have no competing interests. 


\section{Consent for publication}

Not applicable.

\section{Ethics approval}

Animal transplantations were approved by the local Animal Welfare Committee of the University of Leipzig and the local governmental authorities (Landesdirektion Sachsen, permit numbers TW 07/08 and TW 48/12).

\section{Publisher's Note}

Springer Nature remains neutral with regard to jurisdictional claims in published maps and institutional affiliations.

\begin{abstract}
Author details
${ }^{1}$ Interdisciplinary Centre for Bioinformatics (IZBI), University of Leipzig, Leipzig, Germany. ${ }^{2}$ Fraunhofer Institute for Cell Therapy and Immunology (IZI), Leipzig, Germany. ${ }^{3}$ Department of Ophthalmology, Mayo Clinic, Rochester, MN, USA. ${ }^{4}$ Paul Flechsig Institute for Brain Research, University of Leipzig, Leipzig, Germany. ${ }^{5}$ Centre for Biological Engineering, Wolfson School, Loughborough University, Loughborough, UK.
\end{abstract}

Received: 2 September 2016 Revised: 29 January 2017 Accepted: 9 March 2017 Published online: 18 April 2017

\section{References}

1. Sethe S, Scutt A, Stolzing A. Aging of mesenchymal stem cells. Ageing Res Rev. 2006:5:91-116.

2. Wang S, Qu X, Zhao RC. Clinical applications of mesenchymal stem cells. J Hematol Oncol. 2012;5:19.

3. Niyibizi C, Li F. Potential implications of cell therapy for osteogenesis imperfecta. Int J Clin Rheumatol. 2009;4:57-66.

4. Undale AH, Westendorf JJ, Yaszemski MJ, Khosla S. Mesenchymal stem cells for bone repair and metabolic bone diseases. Mayo Clin Proc. 2009;84:893-902.

5. Volarevic V, Arsenijevic N, Lukic ML, Stojkovic M. Concise review: Mesenchymal stem cell treatment of the complications of diabetes mellitus. Stem Cells. 2011;29:5-10.

6. Barbash IM, Chouraqui P, Baron J, Feinberg MS, Etzion S, et al. Systemic delivery of bone marrow-derived mesenchymal stem cells to the infarcted myocardium: feasibility, cell migration, and body distribution. Circulation. 2003;108:863-8.

7. Connick P, Kolappan M, Crawley C, Webber DJ, Patani R, et al. Autologous mesenchymal stem cells for the treatment of secondary progressive multiple sclerosis: an open-label phase 2a proof-of-concept study. Lancet Neurol. 2012;11:150-6.

8. Wyles CC, Houdek MT, Behfar A, Sierra RJ. Mesenchymal stem cell therapy for osteoarthritis: current perspectives. Stem Cells Cloning. 2015;8:117-24.

9. Shi M, Liu ZW, Wang FS. Immunomodulatory properties and therapeutic application of mesenchymal stem cells. Clin Exp Immunol. 2011;164:1-8.

10. Pulavendran S, Vignesh J, Rose C. Differential anti-inflammatory and anti-fibrotic activity of transplanted mesenchymal vs. hematopoietic stem cells in carbon tetrachloride-induced liver injury in mice. Int Immunopharmacol. 2010;10:513-9.

11. Johnson AA, Naaldijk Y, Hohaus C, Meisel HJ, Krystel I, et al. Protective effects of alpha phenyl-tert-butyl nitrone and ascorbic acid in human adipose derived mesenchymal stem cells from differently aged donors. Aging. 2016;9(2):340-52.

12. Johnson AA, Riehle MA. Resveratrol fails to extend life span in the mosquito Anopheles stephensi. Rejuvenation Res. 2015;18:473-8.

13. Naaldijk Y, Johnson AA, Ishak S, Meisel HJ, Hohaus C, et al. Migrational changes of mesenchymal stem cells in response to cytokines, growth factors, hypoxia, and aging. Exp Cell Res. 2015;338:97-104.

14. Kean TJ, Lin P, Caplan Al, Dennis JE. MSCs: delivery routes and engraftment, celltargeting strategies, and immune modulation. Stem Cells Int. 2013;2013:732742.

15. Devine SM, Cobbs C, Jennings M, Bartholomew A, Hoffman R. Mesenchymal stem cells distribute to a wide range of tissues following systemic infusion into nonhuman primates. Blood. 2003;101:2999-3001.

16. Kawada H, Fujita J, Kinjo K, Matsuzaki Y, Tsuma M, et al. Nonhematopoietic mesenchymal stem cells can be mobilized and differentiate into cardiomyocytes after myocardial infarction. Blood. 2004;104:3581-7.

17. Nagaya $N$, Fujii $T$, Iwase $T$, Ohgushi $H$, Itoh $T$, et al. Intravenous administration of mesenchymal stem cells improves cardiac function in rats with acute myocardial infarction through angiogenesis and myogenesis. Am J Physiol Heart Circ Physiol. 2004;287:H2670-6.
18. Jiang W, Ma A, Wang T, Han K, Liu Y, et al. Intravenous transplantation of mesenchymal stem cells improves cardiac performance after acute myocardial ischemia in female rats. Transpl Int. 2006;19:570-80.

19. Morigi M, Introna M, Imberti B, Corna D, Abbate M, et al. Human bone marrow mesenchymal stem cells accelerate recovery of acute renal injury and prolong survival in mice. Stem Cells. 2008;26:2075-82.

20. Deak E, Ruster B, Keller L, Eckert K, Fichtner I, et al. Suspension medium influences interaction of mesenchymal stromal cells with endothelium and pulmonary toxicity after transplantation in mice. Cytotherapy. 2010;12:260-4.

21. Kraitchman DL, Tatsumi M, Gilson WD, Ishimori T, Kedziorek D, et al. Dynamic imaging of allogeneic mesenchymal stem cells trafficking to myocardial infarction. Circulation. 2005;112:1451-61.

22. Ha S, Ahn S, Kim S, Joo Y, Chong YH, et al. In vivo imaging of human adipose-derived stem cells in Alzheimer's disease animal model. J Biomed Opt. 2014;19:051206.

23. Sohni A, Verfaillie CM. Mesenchymal stem cells migration homing and tracking. Stem Cells Int. 2013:2013:130763.

24. Marquez-Curtis LA, Janowska-Wieczorek A. Enhancing the migration ability of mesenchymal stromal cells by targeting the SDF-1/CXCR4 axis. BioMed Res Int. 2013;2013:561098.

25. Karp JM, Leng Teo GS. Mesenchymal stem cell homing: the devil is in the details. Cell Stem Cell. 2009;4:206-16.

26. Sasaki M, Abe R, Fujita $Y$, Ando S, Inokuma D, et al. Mesenchymal stem cells are recruited into wounded skin and contribute to wound repair by transdifferentiation into multiple skin cell type. J Immunol. 2008;180:2581-7.

27. Kidd S, Spaeth E, Dembinski JL, Dietrich M, Watson K, et al. Direct evidence of mesenchymal stem cell tropism for tumor and wounding microenvironments using in vivo bioluminescent imaging. Stem Cells. 2009;27:2614-23.

28. Kang SK, Shin IS, Ko MS, Jo JY, Ra JC. Journey of mesenchymal stem cells for homing: strategies to enhance efficacy and safety of stem cell therapy. Stem Cells Int. 2012;2012:342968.

29. Son BR, Marquez-Curtis LA, Kucia M, Wysoczynski M, Turner AR, et al. Migration of bone marrow and cord blood mesenchymal stem cells in vitro is regulated by stromal-derived factor-1-CXCR4 and hepatocyte growth factor-c-met axes and involves matrix metalloproteinases. Stem Cells. 2006;24:1254-64.

30. Ruster B, Gottig S, Ludwig RJ, Bistrian R, Muller S, et al. Mesenchymal stem cells display coordinated rolling and adhesion behavior on endothelial cells. Blood. 2006:108:3938-44.

31. Segers VF, Van Riet I, Andries LJ, Lemmens K, Demolder MJ, et al. Mesenchyma stem cell adhesion to cardiac microvascular endothelium: activators and mechanisms. Am J Physiol Heart Circ Physiol. 2006;290:H1370-7.

32. Xiao Q, Wang SK, Tian H, Xin L, Zou ZG, et al. TNF-alpha increases bone marrow mesenchymal stem cell migration to ischemic tissues. Cell Biochem Biophys. 2012:62:409-14.

33. Bustos ML, Huleihel L, Kapetanaki MG, Lino-Cardenas $C L$, Mroz L, et al. Aging mesenchymal stem cells fail to protect because of impaired migration and antiinflammatory response. Am J Respir Crit Care Med. 2014; 189:787-98.

34. Scutt N, Johnson AA, Scutt A, Stolzing A. Tissue-specific ageing of rat tendon-derived progenitor cells. J Stem Cell Res Ther. 2015;5:309. doi:10. 4172/2157-7633.1000309.

35. Lopez-Otin C, Blasco MA, Partridge L, Serrano M, Kroemer G. The hallmarks of aging. Cell. 2013;153:1194-217.

36. Rohani L, Johnson AA, Arnold A, Stolzing A. The aging signature: a hallmark of induced pluripotent stem cells? Aging Cell. 2014;13:2-7.

37. Duscher D, Rennert RC, Januszyk M, Anghel E, Maan ZN, et al. Aging disrupts cell subpopulation dynamics and diminishes the function of mesenchymal stem cells. Sci Rep. 2014;4:7144.

38. Li L, Guo Y, Zhai H, Yin Y, Zhang J, et al. Aging increases the susceptivity of MSCs to reactive oxygen species and impairs their therapeutic potency for myocardial infarction. PLoS One. 2014:9:e111850.

39. Borodkina A, Shatrova A, Abushik P, Nikolsky N, Burova E. Interaction between ROS dependent DNA damage, mitochondria and p38 MAPK underlies senescence of human adult stem cells. Aging. 2014;6:481-95.

40. Bach M, Schimmelpfennig C, Stolzing A. Influence of murine mesenchymal stem cells on proliferation, phenotype, vitality, and cytotoxicity of murine cytokine-induced killer cells in coculture. PLoS One. 2014;9:e88115.

41. Dobson KR, Reading L, Haberey M, Marine X, Scutt A. Centrifugal isolation of bone marrow from bone: an improved method for the recovery and quantitation of bone marrow osteoprogenitor cells from rat tibiae and femurae. Calcif Tissue Int. 1999;65:411-3. 
42. Sekiya I, Larson BL, Smith JR, Pochampally R, Cui JG, et al. Expansion of human adult stem cells from bone marrow stroma: conditions that maximize the yields of early progenitors and evaluate their quality. Stem Cells. 2002;20:530-41.

43. Naaldijk Y, Jager C, Fabian C, Leovsky C, Bluher A, et al. Effect of systemic transplantation of bone marrow-derived mesenchymal stem cells on neuropathology markers in APP/PS1 Alzheimer mice. Neuropathol Appl Neurobiol. 2016. [Epub ahead of print].

44. Ito D, Imai Y, Ohsawa K, Nakajima K, Fukuuchi Y, et al. Microglia-specific localisation of a novel calcium binding protein, Iba1. Brain Res Mol Brain Res. 1998:57:1-9.

45. Grudzinska MK, Kurzejamska E, Bojakowski K, Soin J, Lehmann MH, et al. Monocyte chemoattractant protein 1-mediated migration of mesenchymal stem cells is a source of intimal hyperplasia. Arterioscler Thromb Vasc Biol. 2013;33:1271-9.

46. Labedz-Maslowska A, Lipert B, Berdecka D, Kedracka-Krok S, Jankowska U, et al. Monocyte chemoattractant protein-induced protein 1 (MCPIP1) enhances angiogenic and cardiomyogenic potential of murine bone marrow-derived mesenchymal stem cells. PLoS One. 2015:10:e0133746.

47. Leovsky C, Fabian C, Naaldijk Y, Jager C, Jang HJ, et al. Biodistribution of in vitro-derived microglia applied intranasally and intravenously to mice: effects of aging. Cytotherapy. 2015;17:1617-26.

48. Johnson AA, Akman K, Calimport SR, Wuttke D, Stolzing A, et al. The role of DNA methylation in aging, rejuvenation, and age-related disease. Rejuvenation Res. 2012;15:483-94.

49. Liu L, Eckert MA, Riazifar H, Kang DK, Agalliu D, et al. From blood to the brain: can systemically transplanted mesenchymal stem cells cross the blood-brain barrier? Stem Cells Int. 2013;2013:435093.

50. Zlokovic BV. The blood-brain barrier in health and chronic neurodegenerative disorders. Neuron. 2008;57:178-201.

51. Dwyer RM, Potter-Beirne SM, Harrington KA, Lowery AJ, Hennessy E, et al. Monocyte chemotactic protein-1 secreted by primary breast tumors stimulates migration of mesenchymal stem cells. Clin Cancer Res. 2007;13:5020-7.

52. Radde R, Bolmont T, Kaeser SA, Coomaraswamy J, Lindau D, et al. Abeta42driven cerebral amyloidosis in transgenic mice reveals early and robust pathology. EMBO Rep. 2006;7:940-6.

53. McLarnon JG. Microglial chemotactic signaling factors in Alzheimer's disease. Am J Neurodegener Dis. 2012;1:199-204.

\section{Submit your next manuscript to BioMed Central and we will help you at every step:}

- We accept pre-submission inquiries

- Our selector tool helps you to find the most relevant journal

- We provide round the clock customer support

- Convenient online submission

- Thorough peer review

- Inclusion in PubMed and all major indexing services

- Maximum visibility for your research

Submit your manuscript at www.biomedcentral.com/submit

) Biomed Central 